\title{
Association of Uric Acid and C-reactive Protein with the Severity of Coronary Artery Disease Using SYNTAX Score and Clinical SYNTAX Score
}

\author{
Yu Xing, MD, ${ }^{1}$ Jing-Tao Guo, MD,${ }^{2}$ Lu-Yue Gai, $M D,{ }^{1}$ Bo Liu, MD,${ }^{2}$ Dong-Lei Luo, $\mathrm{MD}^{2}$ \\ ${ }^{1}$ Department of Cardiovascular, Chinese PLA General Hospital, Chinese PLA Medical School, Beijing, China; ${ }^{2}$ Department of \\ Cardiovascular, Chengde Central Hospital, Chengde, China
}

\section{ABSTRACT}

Background: The SYNTAX score (SXscore), an anatomical-based scoring tool reflecting the complexity of coronary anatomy, has been associated with the mortality and prognosis of coronary artery disease (CAD). Clinical SYNTAX score (CSS), incorporating clinical factors further augmented the utility of the SXscore to longer-term risk. C-reactive protein (CRP) is related to SXscore. Serum uric acid (UA) is associated with atherosclerosis and CAD. However, serum uric acid combined with CRP may better predict the SXscore and CSS.

Methods: A total of 208 patients (mean age $57.82 \pm 9.39$ years) with chest pain were included in this study. All selected subjects underwent coronary artery angiography and blood test. The relationship between serum UA, CRP and SXscore, and CSS were analyzed.

Results: Age and CRP had a positive correlation with SXs and CSS. DM and fasting glucose correlated with SXscore and CSS respectively. In multivariate regression, serum UA, age, fasting glucose, and body mass index (BMI) were significant discriminant factors of high CSS. The predictive accuracy of CRP for SXscore $>0$ and high CSS using receiver operator characteristic curves was set at the cut off point of $0.205 \mathrm{mg} / \mathrm{dL}$ and $0.145 \mathrm{mg} / \mathrm{dL}$ respectively, (sensitivity $70.9 \%$ and $98 \%$, specialty $48 \%$ and $23.2 \%$ ).

Conclusion: Serum CRP is correlated with SXscore and CSS, serum UA is independently associated with CSS. CRP predicts high CSS at a lower level than it predicts SXscore. Thus, serum CRP combined with serum UA may be useful to predict SXscore and CSS.

\section{INTRODUCTION}

The SYNTAX score (SXscore), an anatomical-based scoring tool reflecting the complexity of coronary anatomy, has been associated with the mortality and prognosis of coronary artery disease (CAD) [Sianos 2005; Capodanno 2009; Serruys

Received Fanuary 20, 2019; accepted March 20, 2019.

This study was supported by Key Medical Research Project of Hebei Province (No.20160296).

Correspondence: Yu Xing, MD, Department of Cardiovascular, Chinese PLA General Hospital, Chinese PLA Medical School, Beijing 100853, China; +86 010 66939553; fax: +8601066939553 (e-mail: docyuxing@126.com).
2009; Serruys 2010; Wykrzykowska 2010]. But SXscore is only an anatomically based tool to assess and grade the angiographic characteristic of coronary lesions quantitatively, so its limitation is its lack of clinical variables which narrows the accuracy of prognosis. SXscore incorporating clinical variables age, creatinine clearance and left ventricular ejection fraction (LVEF) (ACEF), called the clinical SYNTAX score (CSS), showed increased reliability to predict the prognosis of CAD patients after coronary intervention [Garg 2010]. Inflammation plays a role in all stages of atherosclerosis. C-reactive protein (CRP) is an important indicator of atherosclerosis and reported to related to SXscore [Yeh 2003; Armani 2005]. Uric acid (UA) is the end product of purine metabolism, hyperuricemia increases the risk of atherosclerosis, and contributes to the occurrence and prognosis of CAD [Chen 2009; Lee 1995; Puig 1999; Chu 2000; Kim 2010; Yildiz 2012]. So serum UA combined CRP may better predict the severity of CAD using SXscore and CSS.

There have been some studies finding that SUA was positively correlated with SYNTAX score [Ekici 2015], and even could be used as a predictor of severe events [Yu 2014]. Our study aimed to investigate the association of serum uric acid and C-reactive protein with the severity of CAD using SXscore and CSS, which would bring more biological indicator to define the disease and scores.

\section{MATERIALS AND METHODS}

\section{Study Patients}

Between November 2015 and February 2017, 208 consecutive patients with chest pain were selected. All selected subjects received coronary artery angiography and blood testing. Exclusive criteria were congenital heart disease, rheumatic heart disease, pulmonary heart disease, thromboembolic disease, severe unstable angina, acute myocardiac infarction, severe heart failure, infectious disease, allergic to iodine contrast medium, age $>85$ years, being on any UA lowering therapy. All participants gave informed consent and the study protocol was approved by the local Ethics Committee.

Hypertension was defined as systolic blood pressure (BP) $>140 \mathrm{mmHg}$ or diastolic $\mathrm{BP}>90 \mathrm{mmHg}$, or use of antihypertensive medications. Smoking status was defined as both current and former use. BMI was calculated by weight divided by height in meters squared. DM was defined as using oral hypoglycemic agents or insulin or controlled with diet; or two 
Table 1. Baseline Demographic Characteristics

\begin{tabular}{lc}
\hline & Patients $(\mathrm{n}=208)$ \\
\hline Age, $y$ & $57.82 \pm 9.39$ \\
BMI, $\mathrm{kg} / \mathrm{m} 2$ & $25.49 \pm 3.25$ \\
Male sex, \% & 75.96 \\
DM, \% & 24.52 \\
Hypertension, \% & 62.5 \\
Smoker, \% & 53.62 \\
Tc, mmol/L & $4.27 \pm 1.07$ \\
Hyperlipidemia, \% & 9.62 \\
Creatinine, ummol/L & $69.84 \pm 19.32$ \\
CrCL, mL/min & $107.66 \pm 33.58$ \\
UA, ummol/L & $314.83 \pm 82.94$ \\
CRP, mg/dL & $0.73 \pm 1.34$ \\
FPG, mmol/L & $6.19 \pm 2.47$ \\
BUN, mmol/L & $5.74 \pm 3.62$ \\
Platelet, $\times 109 / L$ & $215.29 \pm 63.58$ \\
LVEF, \% & $55.62 \pm 6.77$ \\
ACEF & $1.30 \pm 1.10$ \\
SYNTAX score & $9.88 \pm 9.96$ \\
Clinical SYNTAX score & $15.90 \pm 28.71$ \\
\hline
\end{tabular}

BMI indicates body mass index; DM, diabetes mellitus; TC, total cholesterol; $\mathrm{CrCL}$, creatinine clearance; UA, uric acid; CRP, C-reactive protein; FPG, fasting glucose; BUN, blood urea nitrogen; LVEF, left ventricular ejection; ACEF, age, creatinine clearance and left ventricular ejection fraction.

fasting glucose level $>125 \mathrm{mg} / \mathrm{dL}$, or $2 \mathrm{~h}$ oral glucose levels $>200 \mathrm{mg} / \mathrm{dL}$.

\section{Coronary Angiography}

Coronary angiography was performed by experienced cardiologists who were blinded to the clinical data. SYNTAX (synergy between percutaneous coronary intervention with TAXUS and cardiac survey) scores were evaluated using the SYNTAX website (www.syntaxscore.com) to estimate the extent and severity of CAD. Each coronary stenosis $\geq 50 \%$ in diameter in each vessel $\geq 1.5 \mathrm{~mm}$ was scored according to a standard score system, and the overall scores were summed to be SYNTAX score (SYNTAX score calculator 2013). SYNTAX score $=0$ represented the absence of coronary artery disease or the presence of a $<50 \%$ lesion in any coronary artery. SYNTAX score $>0$ represented the presence of $\geq 50 \%$ lesion in any coronary artery disease.

\section{Clinical SYNTAX Score}

CSS was calculated retrospectively using the formula CSS $=($ SYNTAX score $) \times($ modified ACEF Score $)$. The modified ACEF Score was calculated based on patients' age, left ventricular ejection fraction and creatinine clearance) [Yildiz

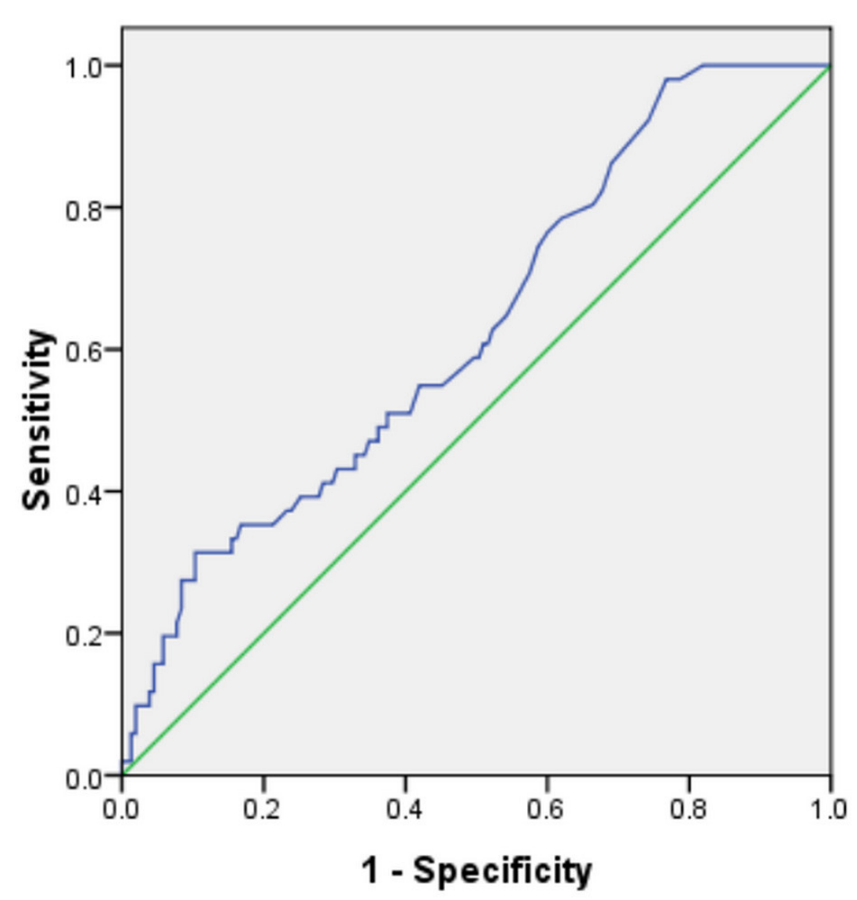

Figure 1. ROC curve analysis of serum CRP levels for discrimination of high CSS. CRP indicates C-reactive protein; CSS, clinical SYNTAX score; AUC, area under the curve; ROC, receiver operating characteristics. CRP >0.145 mg/dL (sensitivity 98\%; specificity 23.2\%; AUC 0.630).

2012]. Creatinine clearance $(\mathrm{CrCl})$ was calculate using the Cockcroft-Gault equation [Cockcroft 1976].

The modified ACEF was calculated retrospectively using the formula $=$ age/left ventricular ejection fraction +1 point for every $10 \mathrm{ml} / \mathrm{min}$ reduction in $\mathrm{CrCl}$ below $60 \mathrm{~mL} / \mathrm{min}$ per $1.73 \mathrm{~m} 2$ (up to maximum of 6 points), the level of $\mathrm{CrCl}$ between $50-59 \mathrm{ml} / \mathrm{min}$ per $1.732 \mathrm{~m}^{2}, 40-49 \mathrm{~mL} / \mathrm{min}$ per $1.732 \mathrm{~m}^{2}, 30-39 \mathrm{ml} / \mathrm{min}$ per $1.732 \mathrm{~m}^{2}$ would receive 1,2 and 3 points, respectively. The LVEF was recorded before the index PCI. The patients were divided into quartiles according to their CSS, defined as control group (CSS $=0$ ), CSS low group (CSS $\leq 15.6)$, CSS mid group (15.6 < CSS < 27.5), CSS high group (CSS $\geq 27.5)$.

\section{Routine Laboratory Measurement}

Blood samples were drawn in all patients in the second morning 6AM-9AM after administration hospital. Laboratory blood measurements included: total cholesterol (Tc), triglycerides (TG), high-density lipoprotein cholesterol (HDL), very-low-density lipoprotein (LDL), CRP, fasting glucose, creatinine, urea nitrogen and UA. Laboratory blood tests were determined by standard methods.

\section{STATISTICAL ANALYSIS}

The data were analyzed with IBM SPSS statistics 17.0 for Windows. Patients' characteristics are summarized as mean \pm standard deviation values or percentages. Logistic regression 
Table 2. Multivariate Logistic Regression Analysis for the Association between High Clinical SYNTAX Score and Uric Acid, Age, Fasting Glucose, BMI

\begin{tabular}{|c|c|c|c|c|c|}
\hline UA, ummol/L & 0.008 & 6.861 & .009 & 1.008 & $1.002-1.014$ \\
\hline FPG, $\mathrm{mmol} / \mathrm{L}$ & 0.186 & 3.672 & .055 & 1.205 & $0.996-1.458$ \\
\hline $\mathrm{BMI}, \mathrm{kg} / \mathrm{m} 2$ & -0.199 & 6.994 & .008 & 0.802 & $0.707-0.950$ \\
\hline
\end{tabular}

UA indicates uric acid; FPG, fasting glucose; BMI, body mass index.

analyses and Spearman correlations were conducted to evaluate associations between serum UA, CRP and SXscore, CSS. Receiver operator characteristic curves were used to detect the discriminate ability of SXscore $>0$ and a high CSS. Statistical significance was set at a two-side $P<.05$.

\section{RESULTS}

\section{Patients' Characteristics}

The mean age of these 208 patients was $57.82 \pm 9.39$ years, $75.96 \%$ of them were male, $24.52 \%$ of them had significant DM, $62.5 \%$ had hypertension, $53.62 \%$ of them were smokers. The details of the clinical characteristics are presented in Table 1.

\section{Association CRP and Uric Acid with the SXscore and CSS}

According to Spearman's analysis, a positive correlation between age, CRP, DM and SYNTAX score was observed $(\mathrm{r}=0.138, P=.048 ; \mathrm{r}=0.219, P=.002 ; \mathrm{r}=0.173, P=.013$, respectively); a significant correlation between age, CRP, fasting glucose and CSS was observed $(\mathrm{r}=0.221, P=.001$; $\mathrm{r}=0.228, P=.001 ; \mathrm{r}=0.165, P=.018$, respectively). There was no correlation of uric acid with SYNTAX score and clinical SYNTAX score $(P>.05)$. UA and CRP had no correlation $(\mathrm{r}=0.036, P=.610)$.

\section{Clinical Factors and SXscore and CSS}

In the binary logistic regression analysis, age and gender were significantly related to SYNTAX score $>0(\mathrm{~B}=1.034$, 95\% CI: $1.321-5.990, P=.007$; B $=0.041,95 \%$ CI: $1.004-$ $1.081, P=.030$, respectively). In the multivariate logistic regression analysis, age, $\mathrm{UA}$ age, fasting glucose, BMI were significantly discriminatory of high clinical SYNTAX score $(\mathrm{OR}=1.008,95 \% \mathrm{CI}: 1.002-1.014, P=.009 ; \mathrm{OR}=1.066$, 95\% CI: $1.014-1.119, P=.012$; OR $=1.205,95 \%$ CI: $0.996-$ $1.458, P=.055 ; \mathrm{OR}=0.802,95 \% \mathrm{CI}: 0.707-0.950, P=.008$, respectively) (Table 2). UA was also the significant discriminator of mid clinical SYNTAX score $(\mathrm{OR}=0.994,95 \% \mathrm{CI}$ : 0.928-1.021, $P=.041)$.

\section{ROC Analysis to Predict SXscore and CSS}

ROC curves tested the sensitivity and specificity of a cutoff value of $0.205 \mathrm{mg} / \mathrm{dL}$ for CRP to predict SYNTAX score $>0$. The areas of under the ROC curve were $(0.596,95 \% \mathrm{CI}$ :
$0.505-0.686, P=.037)$, with $70.9 \%$ sensitivity and $48.1 \%$ specificity (Figure 2). The ROC curves analysis to predict the high clinical SYNTAX score $(\mathrm{CSS} \geq 27.5)$ are presented in Figure 1; the cut off values of age, BUN, fasting glucose, CRP are $60.5 \mathrm{y}, 5.85 \mathrm{mmol} / \mathrm{L}, 5.75 \mathrm{mmol} / \mathrm{L}, 0.145 \mathrm{mg} / \mathrm{dL}$, respectively. Sensitivity as a discriminator of high CSS was $54.9 \%, 51 \%, 54.9 \%$, 98\%, respectively while the specificity was $78.6 \%, 66.5 \%, 79.9 \%, 23.2 \%$, respectively (Table 3).

\section{DISCUSSION}

Inflammation is known to play a pivotal role in progression of atherosclerosis and subsequent cardiovascular disease. Studies have confirmed that the inflammatory protein CRP is an important indicator of atherosclerosis and accepted as an independent risk factor for atherosclerosis, regulating atherosclerotic disease at a fundamental level [Libby 2002; Labarrere 2004]. As an inflammatory marker, CRP has the advantage of wide availability, low cost and ease of use [Sun 2005]. The elevation of CRP is associated with the extent and severity of CAD [Taniguchi 2005]. In agreement in our study, a positive correlation between CRP and SXscore and CSS was observed. Some studies showed that high sensitive CRP (hsCRP) was associated with SYNTAX score in stable coronary artery disease and acute coronary syndrome [Capodanno 2011; Girasis 2011; Wykrzykowska 2011; Pan 2015; Karadeniz 2015; Tanveer 2016; Kurtul 2017]. Our study showed CRP predicts high CSS at a lower level than predicts SXscore. A cut off value of ROC to predict SXscore and CSS was $0.205 \mathrm{mg} / \mathrm{dL}$ and $0.145 \mathrm{mg} / \mathrm{dL}$, respectively. Our study found similar results; CRP predicts CSS better than do Sxscore. It may be that SXscore is only an anatomically based tool to assess and grade the angiographic characteristic of coronary lesions quantitatively, so its limitation is lack of clinical variables, which limits the accuracy of prognosis. Clinical variables such as age, creatinine clearance and left ventricular ejection fraction (LVEF) (ACEF) combined SYNTAX score which called the CSS may be more reliable to predict the prognosis of CAD patients after coronary intervention [Garg 2010; Capodanno 2011; Girasis 2011; Wykrzykowska 2011]. Our study showed that elevated CRP levels is associated with the extent and severity of CAD. CRP may be more sensitive to discriminate high CSS than does 


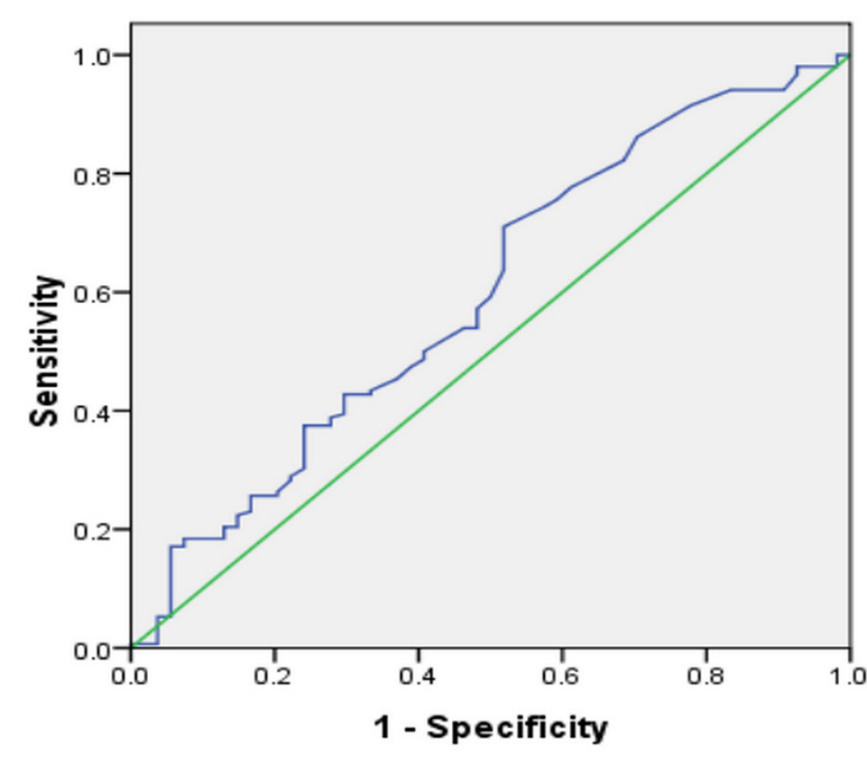

Figure 2. ROC curve analysis of serum CRP levels for discrimination of SYNTAX score $>0$. CRP $>0.205 \mathrm{mg} / \mathrm{dL}$ (sensitivity $70.9 \%$; specificity 48.1\%; AUC 0.596).

SXscore $>0$; this need further study.

Epidemiological evidence suggest that hyperuricemia and cardiovascular disease are highly related [Garg 2010; Kaya 2010; Sinan Deveci 2010; Xiong 2011], increased UA level is an independent and statistically significant predictor of cardiovascular disease and cardiovascular mortality [Freedman 1995; Dutta 2013; Lin 2013]. Our study showed that UA with fasting glucose and BMI were the predictors of high clinical SYNTAX score $(\mathrm{OR}=1.066,95 \%$ CI: $1.014-1.119, P=$ $.012 ; \mathrm{OR}=1.205,95 \%$ CI: 0.996-1.458, $P=.055 ; \mathrm{OR}=0.802$, $95 \%$ CI: $0.707-0.950, P=.008)$. Uric acid was also the significant discriminator of mid clinical SYNTAX score (OR = 0.994, 95\% CI: $0.928-1.021, P=.041)$. Our results indicated that serum UA level was associated with the complexity and severity of CAD and an independent predictor of high CSS. UA is the end product of purine metabolism and can repeatedly act as an antioxidant [Ruggiero 2006]. The rise of serum UA levels suggests a leading risk of cardiovascular disease. However, the causal mechanisms linking elevated UA levels to cardiovascular disease are still unsettled. A popular explanation for how UA might potentially contribute to cardiovascular disease include its ability to induce systemic inflammation. Physiological concentration of serum UA displayed anti-inflammatory and chondroprotective effects [Lai 2017] and serum UA is an important non-enzymatic antioxidant in peripheral circulation. High levels of UA are suggested to provide antioxidant defence in the human body [Davies 1986]. But abnormal high level of UA is often associate with gout, metabolic syndrome, insulin resistance, hypertension, DM and chronic renal disease. In individuals with obesity, glucose intolerance, renal disease, hyperlipidemia, atherosclerosis and hypertension, it was shown that increase of the serum UA level played a part in the pathogenesis of CAD [Lee 1995;
Table 3. Areas Under ROC to Predict High Clinical SYNTAX Score

\begin{tabular}{lccc}
\hline Variables & Area & Sig & $95 \% \mathrm{Cl}$ \\
\hline Age, $y$ & 0.625 & 0.007 & $0.536-0.716$ \\
BUN, mmol/L & 0.599 & 0.030 & $0.508-0.694$ \\
FPG, mmol/L & 0.592 & 0.048 & $0.500-0.688$ \\
CRP, mg/dL & 0.630 & 0.044 & $0.546-0.716$ \\
\hline
\end{tabular}

BUN indicates blood urea nitrogen; FPG, fasting glucose; CRP, C-reactive protein.

Puig 1999; Chu 2000; Kim 2010; Yildiz 2012]. Serum UA was associated with the severity of CAD using Sxscore and CSS, that may be a series of anti-inflammatory effects of UA.

Earlier studies suggested that higher serum UA levels were positively and independently associated with circulating hs-CRP in healthy postmenopausal women [Raeisi 2017]. In the present study, no correlation between serum CRP and serum UA was found $(\mathrm{r}=0.036, P=.610)$. This may be because patients were selected differently or that high serum UA levels were the consequence of various factors, such as inflammation, hypertension, metabolic syndrome. Alternatively, they might be involved in the early phases of vascular damage; or hyperuricemia might induce the expression of hepatic inflammatory molecules by activating the proinflammatory NF-B signaling cascade [Spiga 2017]. Thus, UA metabolism may be a so-called double-edged sword with regards to the inflammatory and/or oxidative response in many organs. Though on the whole, its harmful effects appear to outweigh the benefits of UA. In this study we assigned serum CRP and UA based on their relationship with SXscore and CSS. Our study showed serum CRP and UA may play roles in atherosclerosis in different stages-both high levels are related to the higher burden of coronary atherosclerosis. In the multivariate logistic regression analysis, age, uric acid, fasting glucose, and BMI were significantly discriminator of high clinical SYNTAX score. UA was also a significant discriminator of mid clinical SYNTAX score, which meant that UA was a significant discriminator of SYNTAX score and CCS. At the same time, according to Spearman's analysis, there was a positive correlation between age, CRP, diabetes and SYNTAX score and a significant correlation between age, CRP, fasting glucose and CSS. That is why we combined the 2 serum detectors of UA and CRP to predict the SYNTAX score and CCS. CRP predict high CSS is in lower level than predict SXscore. We preferred to take our study as a primary attempt to call for more research to verify the predictive roles and potential mechanisms of serum $\mathrm{CRP}$ combined UA in predicting the severity of CAD.

\section{Study Limitations}

Patients undergoing coronary angiography who visit a single center do not represent the whole community. Large-scale prospective studies are needed to obtain further information. 


\section{Conclusion}

CRP is correlated with SXscore and CSS which related to complexity and severity of coronary artery lesions; serum UA is independently associated with CSS. CRP is much sensible to predict CSS than do SXscore. Serum CRP and UA may play roles in atherosclerosis in different stages, high levels of both are related to the higher burden of coronary atherosclerosis. Thus, elevated serum CRP combined with elevated serum UA may be useful to identify patients with high coronary atherosclerotic burden.

\section{Acknowledgements}

The authors thank the cardiovascular team of Chengde Central Hospital; without their collaboration, this article would not have been written. We also thank the laboratory staff at Chengde Central Hospital.

\section{REFERENCES}

Armani A, Becker RC. 2005. The biology, utilization and attenuation of C-reactive protein in cardiovascular disease: part I. Am Heart J 149:971-6.

Capodanno D, Caggegi A, Miano M, et al. 2011. Global risk classification and clinical SYNTAX (Synergy between Percutaneous Coronary Intervention with TAXUS and Cardiac Surgery) score in patients undergoing percutaneous or surgical left main revascularization. J Am Coll Cardiol Interv 4:287-97.

Capodanno D, Di Salvo ME, Cincotta G, Miano M, Tamburino C, Tamburino C. 2009. Usefulness of the SYNTAX score for predicting clinical outcome after percutaneous coronary intervention of unprotected left main coronary artery disease. Circ Cardiovasc Interv 2:302-8.

Chen JH, Chuang SY, Chen HJ, Yeh WT, Pan WH. 2009. Serum uric acid level as an independent risk factor for all-cause, cardiovascular, and ischemic stroke mortality: a Chinese cohort study. Arthritis Rheum 61:225-32.

Chu NF, Wang DJ, Liou SH, Shieh SM. 2000. Relationship between hyperuricemia and other cardiovascular disease risk factors among adult males in Taiwan. Eur J Epidemiol 16:13-7.

Cockcroft DW, Gault MH. 1976. Prediction of creatinine clearance from serum creatinine. Nephron 16:31-41.

Davies KJ, Sevanian A, Muakkassah-Kelly SF, Hochstein P. 1986. Uric acid-iron ion complexes. A new aspect of the antioxidant functions of uric acid. Biochem J 235:747-54.

Dutta A, Henley W, Pilling LC, Wallace RB, Melzer D. 2013. Uric acid measurement improves prediction of cardiovascular mortality in later life. J Am Geriatr Soc 61:319-26.

Ekici B, Kütük U, Alhan A, Töre HF. 2015. The relationship between serum uric acid levels and angiographic severity of coronary heart disease. Kardiol Pol 73:533-8.

Freedman DS, Williamson DF, Gunter EW, Byers T. 1995. Relation of serum uric acid to mortality and ischaemic heart disease. The NHANES I Epidemiologic Follow-up Study. Am J Epidemiol 141:637-44.

Garg S, Sarno G, Garcia-Garcia HM, et al. 2010. A new tool for the risk stratification of patients with complex coronary artery disease: the Clinical SYNTAX Score. Circ Cardiovasc Interv 3:317-26.

Girasis C, Garg S, Räber L, et al. 2011. SYNTAX score and Clinical
SYNTAX score as predictors of very long-term clinical outcomes in patients undergoing percutaneous coronary interventions: a substudy of SIRolimus-eluting stent compared with pacliTAXel-eluting stent for coronary revascularization (SIRTAX) trial. Eur Heart J 32:3115-27.

Karadeniz M, Duran M, Akyel A, et al. 2015. High sensitive CRP level is associated with intermediate and high syntax score in patients with acute coronary syndrome. Int Heart J 56:377-80.

Kaya EB, Yorgun H, Canpolat U, et al. 2010. Serum uric acid levels predict the severity and morphology of coronary atherosclerosis detected by multidetector computed tomography. Atherosclerosis 213:178-83.

Kim SY, Guevara JP, Kim KM, Choi HK, Heitjan DF, Albert DA. 2010. Hyperuricemia and coronary heart disease: a systematic review and metaanalysis. Arthritis Care Res (Hoboken) 62:170-80.

Kurtul A, Elcik D. 2017. Procalcitonin is an independent predictor for coronary atherosclerotic burden in patients with stable coronary artery disease. Int J Cardiol 236:61-4.

Labarrere CA, Zaloga GP. 2004. C-reactive protein: from innocent bystander to pivotal mediator of atherosclerosis. Am J Med 117:499-507.

Lai JH, Luo SF, Hung LF, et al. 2017. Physiological concentrations of soluble uric acid arechondroprotective and anti-inflammatory. Sci Rep 7:2359.

Lee J, Sparrow D, Vokonas PS, Landsberg L, Weiss ST. 1995. Uric acid and coronary heart disease risk: evidence for a role of uric acid in the obesity-insulin resistance syndrome: the normative aging study. Am J Epidemiol 142:288-94.

Libby P, Ridker PM, Maseri A. 2002. Inflammation and atherosclerosis. Circulation 105:1135-43.

Lin GM, Li YH, Zheng NC, et al. 2013. Serum uric acid as an independent predictor of mortality in high-risk patients with obstructive coronary artery disease: a prospective observational cohort study from the ET-CHD registry, 1997-2003. J Cardiol 61:122-7.

Pan HC, Sheu WH, Lee WJ, et al. 2015. Coronary severity score and C-reactive protein predict major adverse cardiovascular events in patients with stable coronary artery disease (from the Taichung CAD study). Clin Chim Acta 445:93-100.

Puig JG, Ruilope LM. 1999. Uric acid as a cardiovascular risk factor in arterial hypertension. J Hypertens 17:869-72.

Raeisi A, Ostovar A, Vahdat K, et al. 2017. Association of serum uric acid with high-sensitivity C-reactive protein in postmenopausal women. Climacteric 20:44-8.

Ruggiero C, Cherubini A, Ble A, et al. 2006. Uric acid and inflammatory markers. Eur Heart J 27:1174-81.

Serruys PW, Onuma Y, Garg S, et al. 2009. Assessment of the SYNTAX score in the Syntax study. Euro Intervention 5:50-6.

Serruys PW, Onuma Y, Garg S, et al. 2010. 5-year clinical outcomes of the ARTS II (Arterial Revascularization Therapies Study II) of the sirolimus-eluting stent in the treatment of patients with multivessel de novo coronary artery lesions. J Am Coll Cardiol 55:1093-101.

Sianos G, Morel MA, Kappetein AP, et al. 2005. The SYNTAX score: an angiographic tool grading the complexity of coronary artery disease. Euro Intervention 1:219-27.

Sinan Deveci O, Kabakci G, Okutucu S, et al. 2010. The association between serum uric acid level and coronary artery disease. Int J Clin Pract 64:900-7.

Spiga R, Marini MA, Mancuso E, et al. 2017. Uric acid is associated with 
inflammatory biomarkers and induces inflammation via activating the

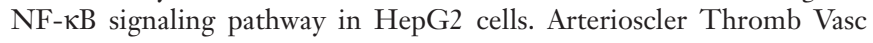
Biol 37:1241-9.

Sun H, Koike T, Ichikawa T, et al. 2005. C-reactive protein in atherosclerotic lesions: its origin and pathological significance. Am J Pathol 167:1139-48.

SYNTAX score calculator. 2013. Available at: http://www.syntaxscore. com. SYNTAX working-group. Accessed May 2018.

Taniguchi H, Momiyama Y, Ohmori R, et al. 2005. Associations of plasma C-reactive protein levels with the presence and extent of coronary stenosis in patients with stable coronary artery disease. Atherosclerosis 178:173-7.

Tanveer S, Banu S, Jabir NR, et al. 2016. Clinical and angiographic correlation of high-sensitivity C-reactive protein with acute ST elevation myocardial infarction. Exp Ther Med 12:4089-98.

Wykrzykowska JJ, Garg S, Girasis C, et al. 2010. Value of the syntax score for risk assessment in the All-Comers population of the randomized multicenter LEADERS trial. J Am Coll Cardiol 56:272-7.

Wykrzykowska JJ, Garg S, Onuma Y, et al. 2011. Value of age, creatinine, and ejection fraction (ACEF score) in assessing risk in patients undergoing percutaneous coronary interventions in the All-Comers LEADERS trial. Circ Cardiovasc Interv 4:47-56.

Xiong Z, Zhu C, Qian X, Zhu J, Wu Z, Chen L. 2011. Predictors of clinical SYNTAX score in coronary artery disease: serum uric acid, smoking, and Framingham risk strati cation. J Invasive Cardiol 23:501-4.

Yeh ET, Willerson JT. 2003. Coming of age of C-reactive protein: using inflammation markers in cardiology. Circulation 107:370-1.

Yildiz A, Kaya Z. 2012. Uric acid: a crucial marker of cardiovascular diseases? Int J Cardiol 159:158.

Yu J, Han J, Mao J. 2014. Association between serum uric acid level and the severity of coronary artery disease in patients with obstructive coronary artery disease. Chin Med J (Engl) 127:1039-45. 